\title{
Connecting the Dots: Mammary Gland and Breast Cancer at Single Cell Resolution
}

\author{
Renée van Amerongen ${ }^{1}$ (1) - Edith C. Kordon ${ }^{2,3}$ (D) $\cdot$ Zuzana Koledova $^{4}$ (i)
}

Received: 13 May 2021 / Accepted: 26 May 2021 / Published online: 14 June 2021

(c) The Author(s), under exclusive licence to Springer Science+Business Media, LLC, part of Springer Nature 2021

Keywords Breast · Mammary gland · Single cell RNA sequencing $\cdot$ Heterogeneity · Dynamics · Imaging

\section{Editorial}

Technological advances drive scientific progress and constantly enable us to push the limits of human knowledge. Following up on the previous successful methods-oriented issue [1], in this special issue entitled Connecting the Dots: Mammary Gland and Breast Cancer at Single Cell Resolution we proudly present a collection of articles on some of the most recent high-resolution techniques, which allow analysis of mammary gland development and disease at the single cell level.

Scientists have longed for the development and implementation of such techniques for decades. The hope is that they will allow us to analyze and dissect the highly dynamic development and tissue remodeling of the mammary gland in full molecular and cellular detail and in real time. This would allow us to closely observe the processes of mammary gland formation, pregnancy, lactation, and involution, as well as the processes of tumor formation and progression. In the past, only partial glimpses into the mammary tissue architecture and spatiotemporal dynamics of gene and protein expression were possible through immunostaining and

Zuzana Koledova

koledova@med.muni.cz

1 Developmental, Stem Cell and Cancer Biology, Swammerdam Institute for Life Sciences, University of Amsterdam, Amsterdam, the Netherlands

2 Instituto de Fisiología, Biología Molecular y Neurociencias (IFIBYNE), CONICET-Universidad de Buenos Aires, Buenos Aires, Argentina

3 Facultad de Ciencias Exactas y Naturales, Departamento de Química Biológica, Universidad de Buenos Aires, Buenos Aires, Argentina

4 Department of Histology and Embryology, Faculty of Medicine, Masaryk University, Brno, Czech Republic in situ hybridization on tissue sections, though the resolution capacity has recently improved through multiplexing techniques [2]. Homogenate-based methods (such as Western and Northern blot analyses, protein arrays, qPCR, bulk RNA sequencing, chromatin immunoprecipitation, etc.) have brought valuable insights into temporal changes in and regulation of gene and protein expression. However, their requirement for pooling cells to achieve sufficient amounts of material have precluded single cell analyses, and these techniques thus offered limited (and increasingly insufficient) spatial resolution. Improvements in this area have been achieved through fluorescence-activated cell sorting or laser-capture microdissection of specific cell population or tissue area of interest. Yet, these methods come short in comparison to the unparalleled resolution and information depth achieved through recently developed and still evolving spatial transcriptomics and single-cell RNA sequencing (scRNA seq) technologies.

In the microscopy world, technical advances have improved spatiotemporal performance of various microscopy modalities and increased computing speeds for processing large imaging data sets, such as high-resolution volumetric imaging data for 3D tissue reconstruction. Novel tissue clearing methods have increased imaging depth achievable by fluorescent imaging of fixed mammary tissue and breast tumor samples as well as whole mammary organoid cultures [3, 4], while imaging windows combined with multiphoton microscopy have enabled long-term imaging of live mammary glands in vivo [4]. In this issue, Messal and colleagues describe how distinct intravital imaging approaches in different genetically engineered mouse strains provide a microscopy toolbox to study mammary gland epithelial dynamics at different developmental stages at single-cell resolution [5]. Using these techniques, they show high motility and continuous intermingling of cells in the terminal end bud, a highly variable contribution of 
single ductal cells to estrous cycle-driven remodeling, and remarkably stable long-term morphology of the mammary gland. Furthermore, their paper discusses important considerations to experimental design and limitations of what is currently technologically possible. Dawson and Visvader take the reader on a microscopic journey too, highlighting the insights that have been obtained from emerging imaging and clearing techniques [6]. This comprehensive review with schematic cartoons should be especially informative for newcomers in the field, as it manages to summarize a wealth of literature and considers both epithelial and non-epithelial cell populations.

While the novel imaging techniques provide improved insights into cellular dynamics, tissue architecture and cell-cell interactions in native tissues, gene expression changes during mammary gland development and disease are being appreciated in unprecedented detail using scRNA seq technology. Advanced bioinformatic analyses of the expression data from thousands of individual cells allow to decipher the genetic programs that drive cell behavior, fate, differentiation dynamics and homeostasis in the mammary gland. This issue also features a unique "behind the scenes" look at the Human Breast Cell Atlas project [7], which aims to generate a comprehensive, spatially resolved atlas of the human breast and the lineage relationships between them. The article relays the experiences of the UK and USA scientists currently involved, and also calls for others in the mammary gland and breast community to join this global effort.

The article by García Solá and colleagues integrates scRNA seq data from three pioneering reports to generate a new comprehensive analysis of postnatal mouse mammary gland development [8]. This approach reveals new cell clusters with particular features and describes the expression profile of individual genes during the pseudo-temporal trajectories of all mammary cell subtypes or the luminal lineages, specifically. The work by Henry et al. expands the collection of scRNA seq datasets for mouse and human, including a cross-species comparison [9], thus providing a rich resource and plenty of room for the reader to ponder how all the information from different datasets and different studies will ultimately fit together. In their perspective article, Martin Carli and colleagues highlight the potential of human milk-derived cells (HMDCs) as a readily available and valuable resource for studying human lactation biology. Showcasing two recent studies, they discuss how scRNA seq of HMDCs can help to unravel the molecular and cellular diversity of secretory mammary epithelial cells in humans, and connect their changes to maternal phenotypes [10].

In summary, this special issue includes original research, review and perspective articles, which we very much enjoyed and also heartily recommend to the reader. While the articles are exclusively focused on normal mammary gland development and function, they provide a wealth of information and important technical considerations that also apply to breast cancer research.

Acknowledgements R.v.A. acknowledges funding from KWF Kankerbestrijding (Dutch Cancer Society, project grant 11082/2017-1) and NWO (Netherlands Science Foundation, VIDI 864.13.002). E.C.K. was funded by CONICET, FONCYT (PICT 2016-2834), University of Buenos Aires, and INC (National Institute of Cancer), Argentina. The work of Z.K. was supported by the Grant Agency of Masaryk University (projects no. MUNI/G/1446/2018 and MUNI/G/1775/2020) and by funds from the Faculty of Medicine MU to junior researcher (Z.K., ROZV/28/LF/2020).

\section{Declarations}

Conflict of Interest The authors declare that they have no conflict of interest.

\section{References}

1. Monkkonen T, Traustadóttir GÁ, Koledova Z. Unraveling the Breast: Advances in Mammary Biology and Cancer Methods. J Mammary Gland Biol Neoplasia. 2020;25:233-6.

2. Mori H, Bolen J, Schuetter L, Massion P, Hoyt CC, VandenBerg S, et al. Characterizing the Tumor Immune Microenvironment with Tyramide-Based Multiplex Immunofluorescence. J Mammary Gland Biol Neoplasia. 2020;25:417-32.

3. Sumbal J, Budkova Z, Traustadóttir GÁ, Koledova Z. Mammary Organoids and 3D Cell Cultures: Old Dogs with New Tricks. J Mammary Gland Biol Neoplasia. 2020;25(4):273-88.

4. Lloyd-Lewis B. Multidimensional Imaging of Mammary Gland Development: A Window Into Breast Form and Function. Front Cell Dev Biol. 2020;8:203.

5. Messal HA, van Rheenen J, Scheele CLGJ. An Intravital Microscopy Toolbox to Study Mammary Gland Dynamics from Cellular Level to Organ Scale. J Mammary Gland Biol Neoplasia. 2021. https://doi.org/10.1007/s10911-021-09487-2.

6. Dawson CA, Visvader JE. The Cellular Organization of the Mammary Gland: Insights From Microscopy. J Mammary Gland Biol Neoplasia. 2021. https://doi.org/10.1007/s10911-021-09483-6.

7. van Amerongen R. Behind the Scenes of the Human Breast Cell Atlas Project. J Mammary Gland Biol Neoplasia. 2021. https:// doi.org/10.1007/s10911-021-09482-7.

8. García Solá ME, Stedile M, Beckerman I, Kordon EC. An Integrative Single-cell Transcriptomic Atlas of the Post-natal Mouse Mammary Gland Allows Discovery of New Developmental Trajectories in the Luminal Compartment. J Mammary Gland Biol Neoplasia. 2021. https://doi.org/10.1007/s10911-021-09488-1.

9. Henry S, Trousdell MC, Cyrill SL, Zhao Y, Feigman MJ, Bouhuis JM, Aylard DA, Siepel A, dos Santos CO. Characterization of gene expression signatures for the identification of cellular heterogeneity in the developing mammary gland. J Mammary Gland Biol Neoplasia. 2021. https://doi.org/10.1007/s10911-021-09486-3.

10. Martin Carli JF, Trahan GD, Rudolph MC. Resolving Human Lactation Heterogeneity Using Single Milk-Derived Cells, a Resource at the Ready. J Mammary Gland Biol Neoplasia. 2021. https://doi. org/10.1007/s10911-021-09489-0.

Publisher's Note Springer Nature remains neutral with regard to jurisdictional claims in published maps and institutional affiliations. 